\title{
Construcción de comunidades hídricas en México
}

\section{Construction of Water Communities in Mexico \\ Construção de comunidades hidricas no México}

\author{
Citlalli Aidee Becerril-Tinoco* \\ Felipe de Alba Murrieta**
}

Recibido: 22 de noviembre de 2013

Aprobado: 17 de marzo de 2014

Doi: dx.doi.org/10.12804/territ30.2014.08

Para citar este artículo:

Becerril-Tinoco, C. A. y De Alba Murrieta, F. (2014). Construcción de comunidades hídricas en México. Territorios, 30, 171-189. doi: dx.doi.org/10.12804/territ30.2014.08

\begin{abstract}
* Licenciada en Geografía y Ordenación del Territorio, Universidad Autónoma del Estado de México. Magister en Evaluación de Impacto Ambiental y Doctorado en Estudios de Desarrollo, Universidad de East Anglia, Reino Unido. Universidad Autónoma Metropolitana. Unidad Iztapalapa, México. Correo electrónico: citlalli_est@yahoo.com

* Magister en Ciencia Política (FCPYS-UNAM), México. Doctor en Planificación Urbana, Universidad de Montreal, Canadá.Profesor-investigador Universidad Autonoma Metropolitana Unidad Cuajimalpa, México. Correo electrónico: dealbamf@gmail.com
\end{abstract}


Palabras clave

Comunidades

imaginadas, agua, empoderamiento, Estadonaturaleza, México.

Keywords

Imagined communities, water, empowerment, State-nature, México.

Palavras-chave

Comunidades imaginadas, água, empoderamento, Estadonatureza, México.

\section{territarias 30}

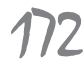

\section{RESUMEN}

A partir de la noción de comunidad hidrica imaginada, parafraseando a Benedict Anderson (1983), en este artículo se estudian casos de comunidades originarias que desarrollan procesos identitarios y formas de legitimación colectiva a través de la apropiación, uso y manejo del recurso agua.

Con esta perspectiva, fue posible observar cómo algunas comunidades localizadas en el Estado de México han obtenido mayor empoderamiento (empowerment) sobre el manejo del agua y han adquirido otras capacidades en la vía de disputar al Estado la dirección, el control, el manejo, el acceso y el cobro del servicio de agua potable. Se concluye que el acceso, la operación y la administración del servicio de agua potable por parte de los comités de aguas, da pauta a la construcción de comunidades hídricas que se asumen participantes de una novedosa relación Estado-naturaleza.

Este artículo se funda en una serie de entrevistas realizadas en los años 2008, 2009 y 2010 en tres comunidades del Estado de México con características similares en sus prácticas de manejo del agua.

\section{ABSTRACT}

Taking into account the concept of imagined water communities elaborated by Benedict Anderson (1983) in this paper, we have studied cases about originary communities that have developed identity processes and collective legitimacy forms through the ownership and property of water resources. Through this perspective it was possible to identify how communities located within the State of Mexico territory have gained power and have had empowerment of water resources. These communities have also gained other capabilities such as control, management, access and the collection of water users payment for the drinking water service they receive. Through imagined communities perspective it also helps to identify identity processes and legitimacy processes within originary communities.

This paper concludes that access, operation and management of the drinking water supply service by water committees is useful to built water communities that become active participants in the relationship State-nature.

This paper was elaborated with information obtained from interviews carried out in 2008, 2009 and 2010 in three communities from the State of Mexico. These communities share similar characteristics in terms of their water management practices.

\section{RESUMO}

A partir da noção de comunidade hídrica imaginada, parafraseando a Benedict Anderson (1983), neste artigo estudam-se casos de comunidades originárias que desenvolvem processos identitários e formas de legitimação coletiva através da apropriação, uso e gestão do recurso água.

Com esta perspectiva, foi possível observar como algumas comunidades localizadas no Estado do México têm obtido maior empoderamento (empowerment) sobre a gestão da água e têm adquirido outras capacidades na via de disputar ao Estado a direção, o controle, a gestão, o acesso e a cobrança do serviço de água potável. Conclui-se que o acesso, a operação e a administração do serviço de água potável por parte dos comitês de águas, dá pauta à construção de comunidades hídricas que se assumem participantes de uma nova relação Estado-natureza. 
Este artigo fundamenta-se em uma série de entrevistas realizadas nos anos 2008, 2009 e 2010 em três comunidades do Estado do México com características similares em suas práticas de gestão da água.

\section{Introducción}

En áreas rurales marginadas de países en desarrollo, principalmente en América Latina, se pueden identificar una gran variedad de prácticas legales o paralegales en el manejo de recursos naturales. Tal es el caso del agua, la cual, se puede encontrar en comunidades originarias organizadas de manera autónoma. Pero, ¿qué entendemos por prácticas paralegales? Aquí se referirá a las diferentes prácticas de control, manejo operación y mantenimiento del servicio de agua potable y la infraestructura que son consideradas válidas (incluyen leyes, reglas y normas), que están fundadas en autoridades legitimadas por usos y costumbres, y que adquieren diferentes formas en el uso histórico de un recurso natural. La naturaleza de los usos y costumbres (también conocidas como prácticas consuetudinarias) son en realidad una estrategia de dichas comunidades para manejar los conflictos tanto con sus miembros como en sus vínculos con el exterior (Von Benda-Beckmann, Benda-Beckman y Spiertz,1998).

En varios países de América Latina los gobiernos nacionales han aprobado políticas que buscan sustituir prácticas consuetudinarias en el manejo de recursos naturales por leyes 'modernas' del Estado-nación. Esto se hace a partir de declarar como propiedad del Estado-nación a los recursos naturales tales como la tierra, el agua y los bosques.

En el caso de México, el gobierno federal estableció dicha 'apropiación' en la Constitución Política desde 1917, aunque fue hasta los años setenta que esto se concretó en una ley específica. El control y explotación del agua por parte del Estado significa, por oposición, que hay una batalla constante por 'ordenar' un gran número de sistemas legales tradicionales, consuetudinarios o indígenas, a los que se busca sustituir por las leyes escritas (Von Benda-Beckmann, Benda-Beckman y Spiertz,1998).

A pesar de ello, en el siglo XXI es posible encontrar diversas prácticas paralegales en comunidades originarias, pequeños pueblos o congregaciones que controlan, a su manera, las aguas (superficiales o subterráneas) localizadas dentro de su territorio. De la misma forma que el Estado lo hace, estas comunidades desarrollan prácticas que están reguladas por instituciones locales y comunitarias reconocidas y legitimadas por su población para mantener el control político y administrativo sobre sus recursos naturales, así como para mantener los derechos para usar y transferir estos recursos. Su propósito no parece entrar en disputa con el Estado, pero en los hechos, lo remplazan.

En la literatura científica se identifican regularmente dos tipos de sistemas legales: formal e informal (Helmke y Levitsky, 
${ }^{1}$ Este artículo es parte de una investigación particular, que ha dado lugar a una tesis de Doctorado en Desarrollo Internacional en la Universidad East Anglia, Gran Bretaña, sobre Gobernabilidad consuetudinaria en tres comunidades mexicanas, así como una investigación general financiada por el PROMEP (SEP-México) sobre la gestión 'informal' del agua en pueblos originarios.

\section{territarias 30} 174
2004; Matsinhe, Juízo, Macheve y Santos, 2008; Von Benda-Beckmann, 1995; Von Benda-Beckmann, Benda-Beckman y Spiertz, 1998; Zwarteveen, Roth y Boelens, 2005). El sistema formal está reconocido por una ley escrita, principalmente creada por el Estado-nación mientras que el sistema informal se caracteriza por leyes 'no escritas' (consuetudinaria), trasmitidas oralmente en ceremonias o en reuniones públicas (Von Benda-Beckmann, BendaBeckman y Spiertz, 1998).

En este artículo se pretende analizar la experiencia de autogobierno de comunidades organizadas por costumbre para administrar el servicio de agua potable. A partir de ello se busca resaltar aspectos de legitimidad y eficacia en el manejo del agua en territorios comunitarios que en los hechos se separan de la gestión federal en el manejo de sus recursos.

\section{Metodología ${ }^{1}$}

La información obtenida para la elaboración de este artículo es resultado del trabajo de campo llevado a cabo en tres comunidades del Estado de México (San Mateo, Santiaguito y San Francisco), las cuales fueron seleccionadas debido a las siguientes características: las tres comunidades se encuentran localizadas en un área rica en agua subterránea; administran el agua de manera autónoma y se organizan por costumbre para legitimar decisiones; a pesar de estar asentadas en una zona rica en agua subterránea, padecen escasez y estrés hídrico debido a problemas causados por la administración del servicio de agua potable.

\section{Localización geográfica del área de estudio}

Las ciudades caso de estudio se localizan en zonas peri-urbanas de los municipios de Almoloya de Juárez y Toluca, ambas en el estado de México. Santiaguito y San Francisco se localizan en el primero y San Mateo, en el segundo. Almoloya de Juárez se localiza al noroeste del municipio de Toluca. El mapa 1 muestra la localización geográfica de las ciudades consideradas en este estudio.

El uso de múltiples casos de estudio permite identificar los patrones similares de estrés hídrico y la comparación de hechos complejos en una zona con abundante agua subterránea; al igual que las decisiones en torno a la dotación de agua potable y los problemas de suficiencia al proveer el servicio de dotación de esta (Pahl-Wostl, 2009); por ejemplo, el tipo de organización de un comité de aguas, principales autoridades que coordinan la administración del agua potable, su disponilibilidad, los pagos por este servicio, los actores involucrados en la provisión, entre otros. Para reducir posibles sesgos en esta investigación fue importante triangular la información obtenida en campo y en fuentes secundarias. La triangulación permite comparar y contrastar los diferentes elementos que interactuan en cada uno de los casos de estudio; asimismo, permite fortalecer la validez de la investigación. No obstante, la información 


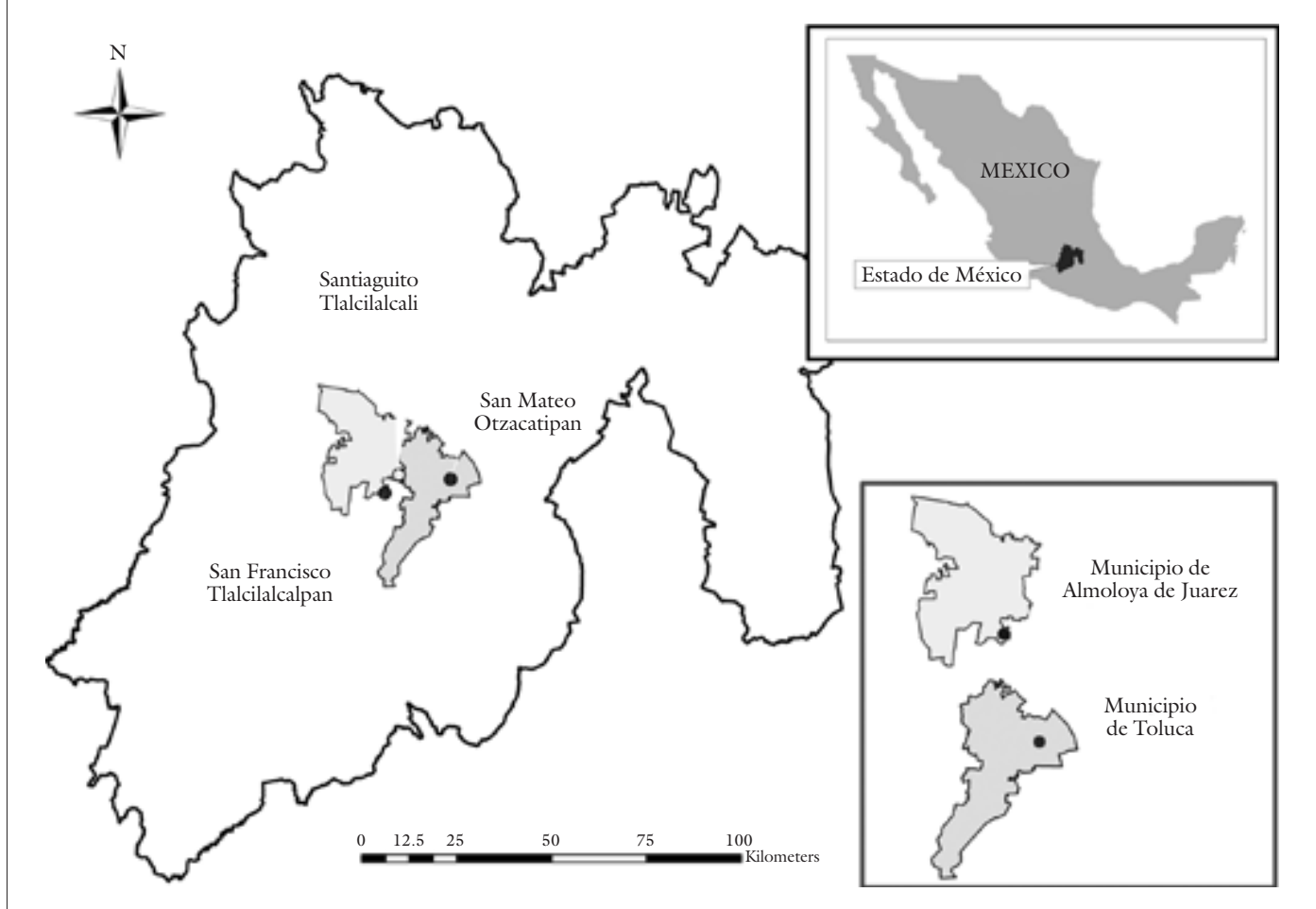

Fuente: Elaboración propia con base en información de campo.

obtenida fue de múltiples casos de estudio, como el representativo de las comunidades que se organizan por costumbre para administrar sus aguas, este trabajo prestará especial atención al caso de la comunidad de Santiaguito.

En este estudio se utilizaron dos técnicas de investigación: entrevistas semiestructuradas y grupos de enfoque, con el fin de contrastar las prácticas paralegales y sus alcances. Una y otra técnica nos permitió dar coherencia a la información recolectada. Las entrevistas semiestructuradas se llevaron a cabo con representantes y ex representantes de gobierno, propietarios de pozos, asuarios domésticos de agua y miembros del comité de aguas de las tres comunidades. Los grupos de enfoque consideraron a los usuarios y a las autoridades del agua de cada comunidad.

\section{¿Qué es una comunidad} hídrica imaginada?

En este artículo se busca estudiar a las comunidades antes mencionadas a partir del 
concepto comunidades hidricas imaginadas que guiará la perspectiva teórica de este artículo. Es decir, se trata de construir dicha noción - originada en el famoso texto de Bennedict Anderson Imagined communities: Reflections on the origins and spread of nationalism (1983) - , a partir de las prácticas informales que remplazan (en los hechos) al Estado y a sus leyes con instituciones comunitarias. Asimismo, se trata de aproximarnos a las formas que emplean las comunidades para reconocer o no las diferentes leyes como un proceso de construcción con el que legitiman o no sus derechos de acceso al agua.

En este artículo, las comunidades hídricas imaginadas son colectivos sociopolíticos con procesos de construcción de nuevas formas de soberanía y se forman a través de redes de lealtad en ámbitos locales, aunque su acción política pueda alcanzar expresiones globales (Appuradai, 2003; Sassen, 2007; Davis, 2010; Devetak y Higgott, 1999; Linklater, 1993).

No obstante que pueden coexistir con el Estado moderno, a veces desarrollan un interés por reemplazarlo: a veces alternativamente, a veces como una consecuencia natural de la maduración política de su organización o por los alcances de su resistencia. Sin embargo, las comunidades hídricas imaginadas no necesariamente tienen que contener en su organización figuras institucionales y ni buscan reemplazar al Estado aunque el interés por involucrarse con este aumente (Anderson, 1983). Esta cualidad es entonces producto del tiempo que tienen en el desarrollo de sus estrategias co- lectivas, que los termina convenciendo de la necesidad de funcionar fuera de los códigos impuestos por el Estado en funciones.

$\mathrm{Al}$ interior de su organización, se crean procesos de legitimidad para cohesionar a su población, lo que les permite adquirir (o desarrollar) culturas de pertenencia, siendo esta su segunda principal característica (Anderson, 1983). Por una parte, este sentido de pertenencia puede facilitar la colaboración entre cada uno de sus miembros, o entre pequeños grupos formados a propósito de ciertos objetivos, o para la complicidad con quienes se establecen 'fuera' del Estado (como el caso de quienes utilizan el agua de pozos particulares para revenderla sin pagar impuestos).

Por otra parte, este sentido de pertenencia si bien es desarrollado por la solidaridad entre sus miembros (acciones de autoorganización), también se puede originar de la vinculación con otras comunidades, sea por la similiaridad de condición que viven, o por procesos identitarios similares (acciones económicas de reproducción o redes de interés mutuo). Dichas redes de pertenencia se manifiestan o se pueden manifestar, una vez más, independientemente de los procesos de legitimación del Estado.

Finalmente, es a través de estas redes de pertenencia o de estos procesos identitarios que podemos encontrar reflejados en dichas comunidades hídricas imaginadas algunas relaciones de poder de interés para este artículo (condición étnica, pobres desplazados por la urbe, condición de género u otras formas de minorías sociales) (Alexander, 2003; Archer, 1996). 


\section{Construcción de comunidades hídricas}

Autores tales como Lynch y Talbott (1995), Von Benda-Beckmann, Benda-Beckman y Spiertz (1998) han hecho estudios sobre comunidades originarias y concluyen que sus derechos y su ley consuetudinaria son un elemento positivo en el manejo de recursos naturales. Estos autores argumentan que el reconocimiento de las reglas creadas y legítimas por esas comunidades son una expresión de valores y necesidades esenciales de su población. Basados en esta perspectiva, sugieren quitar de las legislaciones formales aquellas medidas que debiliten o contradigan la organización y formas de manejo comunitario de recursos naturales. Sin embargo, esta idea parece complicada llevarla a la práctica puesto que la validez y reconocimiento de leyes no escritas (informales) tienen una influencia limitada, casi siempre en zonas rurales, donde una comunidad ejerce sus tradiciones e identidad.

En este sentido, los actores que administran un recurso natural se enfrentan a un sistema legal único aunque con varios niveles de operación (federal, estatal, municipal), además tienen que coexistir con diversos sistemas normativos y derechos de control, gestión y operación, especialmente cuando se trata del agua. Por ejemplo, existen sistemas regidos por el Estado, privados y socialmente constituidos.

Cuando se trata del manejo del agua, la comunidad genera, acepta o rechaza las reglas que les permiten interactuar con la población que la integra. La construcción de una comunidad hídrica ocurre a partir de varias razones, tres de ellas han sido identificadas por Helmke y Levitsky (2004).

Primero, debido a que las instituciones formales están incompletas (o son ineficaces), es decir, no pueden cubrir todos los eventos, servicios y conflictos; entonces, son creadas reglas informales para hacer frente a los problemas no cubiertos por aquellas. Segundo, en situaciones particulares, las instituciones informales pueden ser una 'segunda mejor opción', para resolver problemas derivados de la ineficacia de las instituciones formales. Bajo estas circunstancias las instituciones informales sustituyen a las formales debido a sus alcances limitados y porque el proceso de elaboración de reglas carece de autoridad. Tercero, en consecuencia, la población prefiere autoorganizarse y crear una institución informal para controlar lo que está o no permitido dentro de la sociedad (Helmke y Levitsky, 2004). Cuarto, las instituciones informales han surgido y se consolidan como parte de la organización tradicional de la comunidad (Becerril-Tinoco, 2012). En este plano, el de la comunidad, se han creado instituciones informales para administrar y manejar recursos como el agua y el servicio de agua potable. Este tipo de estructura organizacional forma parte de la participación por costumbre, que asumen los miembros de comunidades con el objetivo de manejar directamente la operación y mantenimiento del servicio de dotación de agua de los pozos que se encuentran en territorio comunitario. El objetivo no es reemplazar todo aquello que les es ajeno: el territarias 30 177 
Estado, sino conservar la propiedad de sus recursos naturales a pesar de las presiones externas y de las limitaciones que tienen para administrar el recurso con eficiencia.

De esta forma, los integrantes del comité de aguas, legitimados por los ciudadanos, deciden administrarlas porque con ello impiden que sean transportadas fuera de su comunidad y que los habitantes padezcan insuficiencia del líquido. La precariedad recibida en el servicio de dotación de agua potable es el objeto que justifica una 'separación' que hay entre instituciones informales y administraciones formales, del Estado. Ello se ve expresado en la opinión de un usuario comunitario del servicio de agua:

Nosotros no queremos que el agua la administre el municipio, preferimos hacerlo nosotros mismos aunque tengamos algunos problemas. Si el municipio entra y le dajamos el control y los derechos del agua, podríamos tener mucha menos agua que la que tenemos ahora porque [el municipio] la repartiría entre más comunidades, mientras que nosotros solo la repartimos entre los que vivimos dentro de ella (usuario doméstico, Santiaguito, abril, 2009).
Un punto central en el estudio de las comunidades hídricas que se gobiernan por costumbre es la obtención de la legitimidad. Es decir, cómo se validan las decisiones comunes para ser reconocidas y aceptadas por la comunidad y se reconocen los derechos para hacer uso de recursos específicos.

\section{Derechos que legitiman los usos del agua}

Schlager y Ostrom (1992) y Ostrom (2001) encontraron que hay derechos de acceso, de extracción, de manejo, de exclusión y de venta o trasferencia, los cuales son reconocidos por los actores en función, tanto del tipo de propiedad que tienen sobre el uso del agua como del reconocimiento que tienen por parte de otros actores. Esta caracterización es clave para reconocer que alrededor de todo ello existe un sistema informal/tradicional/por costumbre, y que ello permite a actores poseer, utilizar y manejar cantidades limitadas de agua. Es decir, existen actores dentro del sistema informal que buscan hacer valer su derecho legal o en acción para tener acceso al agua (ver figura 1).

Figura 1. Acceso al agua en un sistema informal 
La interacción de los actores involucrados en un sistema de agua y los tipos de derechos que pueden poseer están en función de la posición jerárquica que ocupan dentro del organigrama. Cada actor tiene derechos específicos según el rol que cumplen: dueños, propietarios, solicitantes, usuarios o acreedores autorizados, por ejemplo.

Recapitulando, para analizar cómo se conforma una institución informal y una comunidad legitima sus decisiones y derechos y los usuarios responden a la organización comunitaria, la siguiente sección analiza tres casos de estudio de comunidades y los derechos de los diferentes actores para hacer uso del agua potable.

\section{Casos de estudio}

En este artículo se estudian los actores involucrados en los procesos de legitimación de las tres comunidades hídricas mencionadas. Aquí se denomina comunidades hídricas a estas tres comunidades del Estado de México, San Francisco, San Mateo y Santiaguito porque: 1) tienen prácticas similares de consumo del agua potable; 2) se organizan por costumbre para gobernar, operar, administrar y mantener el servicio de agua potable; 3 ) se organizan a través de un comité de aguas, responsable de mantener el control tanto del agua subterránea como de la infraestructura para su distribución; 4) obtienen el agua potable de pozos que se encuentran localizados dentro del territorio comunitario; 5 ) se encuentran sobre un área rica en recursos hídricos; 6) a pesar de la riqueza hídrica de la zona, los consumidores frecuentemente no reciben el servicio de dotación de agua potable que les permite cubrir necesidades básicas, lo que les hace buscar nuevas formas de adaptación para obtener el agua necesaria; 7) aunque cada comité de aguas representa la forma tradicional de organización del agua, sus miembros tienen poca experiencia en el manejo del servicio. ${ }^{2}$

En un sistema comunitario basado en la costumbre interactúan múltiples actores con diferentes responsabilidades, necesidades y formas de aprovechar el agua. En la gestión y dotación de agua para uso doméstico se han identificado diversos actores.

\section{Derechos de propiedad sobre el agua: elementos que legitiman su uso}

Los actores involucrados en sistemas tradicionales han sido identificados por Schlager y Ostrom (1992) y Ostrom (2001), en México se encontró una categoría más que son los usuarios no autorizados (tabla 1).

En esta clasificación entran los usuarios domésticos que no pagan por el derecho de acceso, pero que se benefician o satisfacen sus necesidades. En esta categoría entran también los vendedores intermediarios, aquellos que compran el agua a un actor privado (propietario de un pozo) y que la revenden a las comunidades que no la reciben con suficiencia.

En comunidades gobernadas por instituciones consuetudianarias con mayor frecuencia son reconocidos aquellos 'derechos
${ }^{2}$ Cada comité es renovado en promedio una vezal año, lo que dificulta el desarrollo de habilidades de administración de este servicio. 
${ }^{3}$ Existen varias categorías cuya función está claramente relacionada con una operación autónoma de la comunidad hidrica. Dueño: posee el derecho de vender o transferir un bien sin que esto afecte el uso que el dueño desea tener sobre el recurso. Propietario: tiene los mismos derechos de un solicitante más el derecho a determinar quién accesa $y$ extrae el recurso. Los propietarios generan reglas para excluir a los que no contribuyen con el pago respectivo. Solicitante: puede poseer derechos de operación, es decir, de acceso, de extracción y de manejo de un recurso, después de entenderse vía un pago otra forma de intercambio, con el dueño. Usuario autorizado: tiene derechos de acceso y extracción. Acreedor autorizado: son aquellos que pagan por usar un recurso, tal como el agua (Schlager y Ostrom, 1992).

${ }^{4}$ Los derechos en acción no son usualmente legitimados. Depende de cada individuo si decide ponerlo en práctica o no en función de sus necesidades y posibilidades de acceso al agua.

\section{territarias 30} 180

Tabla 1. Derechos sobre el agua asociados a la posición de los actores en el sistema de derechos de propiedad

\begin{tabular}{|l|c|c|c|c|c|c|}
\hline $\begin{array}{c}\text { Posición } \\
\text { del actor }\end{array}$ & $\begin{array}{c}\text { Dueño: } \\
\text { Estado } \\
\text { De precho } \\
\text { dad (PRS) } \\
\text { nación a } \\
\text { través de } \\
\text { Conagua }\end{array}$ & $\begin{array}{c}\text { Propieta- } \\
\text { rio: estado, } \\
\text { municipio, } \\
\text { comunidad, } \\
\text { propietario } \\
\text { local de un } \\
\text { pozo de } \\
\text { agua }\end{array}$ & $\begin{array}{c}\text { Solicitante } \\
\text { autorizado } \\
\text { de agua: IF: } \\
\text { Organismo } \\
\text { descentra- } \\
\text { lizado, IC: } \\
\text { comités de } \\
\text { agua }\end{array}$ & $\begin{array}{c}\text { Usuario } \\
\text { autori- } \\
\text { zado: } \\
\text { pipero/ } \\
\text { bombero }\end{array}$ & $\begin{array}{c}\text { Ingreso/acree- } \\
\text { dor autorizado al } \\
\text { agua proveniente } \\
\text { de acuíferos o al } \\
\text { servicio de agua } \\
\text { potable: dueño de } \\
\text { la casa, usuario } \\
\text { doméstico de agua }\end{array}$ & $\begin{array}{c}\text { Usuario no autorizado: } \\
\text { usuarios domésticos de } \\
\text { agua quienes no pagan } \\
\text { por derecho a accesar y } \\
\text { extraer agua subterránea: } \\
\text { intermediarios, vendedo- } \\
\text { res de agua (transporta- } \\
\text { da en pipas) }\end{array}$ \\
\hline Acceso & IF/IC & IF/IC & IF/IC & IC & IF/IC & IF/IC \\
\hline Extracción & IF/IC & IF/IC & IC & IC & & IF/IC \\
\hline Manejo/gestión & IF/IC & IF/IC & IF/IC & & & IC \\
\hline Exclusión & IF/IC & IF/IC & & & & \\
\hline Venta/ & IF/IC & IC & IC & & & \\
transferencia & IF & & & & \\
\hline
\end{tabular}

IF: Derechos de propiedad sobre el agua de las instituciones oficiales/formales, reconocidas por las leyes mexicanas escritas y las instituciones oficiales del agua.

IC: Derechos de propiedad sobre el agua reconocidos por las instituciones consuetudinarias o informales.

Fuente: Adaptado de Schlager y Ostrom (1992) y Ostrom (2001) con la información obtenida en campo.

en acción' (Boelens, 2008), ${ }^{4}$ que no son legitimados por una autoridad, pero que sí pueden ser reconocidos por los usuarios y que además permiten a los usuarios no autorizados tener acceso al agua. Los derechos en acción se definen también como el uso sin autorización, formal o informal, de un recurso. En este sentido, son las relaciones sociales que se desarrollan en un territorio y que son normalmente aceptados por usos y costumbres. Enseguida se profundiza sobre los actores no autorizados porque forman parte de una realidad vigente en las comunidades de estudio.

Cuando se habla de los usuarios no autorizados, nos referimos a aquellos que no pagan por el derecho de acceso al agua.
Esta categoría incluye tanto a usuarios domésticos que no pagan por el agua que consumen, como a los vendedores de agua que no pagan por el derecho de acceso y de extracción de agua a la institución respectiva. Por ejemplo, en la comunidad de Santiaguito se identificaron vendedores de agua en pipa que no pagan impuestos o permisos de extracción ni los derechos respectivos a la Comisión Nacional del Agua (Conagua) o a una institución local, por ejemplo, Agua y Saneamiento de Toluca (AysT) o la Comisión de Agua del Estado de México.

En el caso de los usuarios domésticos de agua no autorizados, el incumplimiento al pago por el servicio puede crear una 
deuda acumulada de varios años consecutivos. Aun así, el no pago por el recurso no necesariamente los limita para acceder a esta. En las comunidades con formas de gobierno consuetudinario hay usuarios que son clasificados según la institución que los identifica. Por ejemplo, pueden ser clasificados como 'usuarios no autorizados' por las instituciones formales de agua, pero son clasificados como 'usuarios autorizados' por las instituciones informales de su comunidad. La autorización que las instituciones informales otorgan, puede estar validada no por el pago del agua, sino por los usos y costumbres de la misma. Dentro de esta categoría se encuentran tanto los usuarios domésticos como los vendedores de agua transportada en pipas.

Algunos vendedores de agua pueden no tener autorización por las instituciones formales de agua, pero igualmente mantienen sus derechos como propietarios sobre el agua cuando son aprobados por instituciones comunitarias. Esto es así porque los vendedores de agua generalmente pagan en efectivo al propietario del pozo el derecho de extracción y acceso al agua subterránea. Una vez que los vendedores de agua transportada en pipa pagan, tienen el permiso legítimo por el propietario del pozo para extraer ya sea 10,15 o 20 mil litros cada vez que hacen un pago, por llenar una pipa de agua y en un día pueden llenar tantas veces como puedan pagar por el agua en función del tiempo y las ventas que tengan. A nivel comunitario, los propietarios de los pozos pueden ser actores privados locales y son ellos junto con los comités de agua y los consumidores quienes legitiman el acceso al agua para fines de venta y transporte.

Debido a que las comunidades estudiadas frecuentemente se enfrentan a problemas de insuficiencia en el servicio, continuamente tienen que recurrir a la compra del líquido en pipas. Bajo estas circunstancias, los miembros de una comunidad reconocen y legitiman el papel que juegan los vendedores de agua para proveer un servicio a través de su venta.

Los organismos públicos descentralizados tal como AysT y Conagua consideran a los vendedores de agua como usuarios ilegales, por tanto, sin autorización para accesar, usar o vender. Esto es porque los vendedores no pagan impuestos ni derechos de uso al gobierno municipal, estatal o federal, pues su principal actividad económica es la reventa de agua subterránea.

Un representante de una institución oficial de agua del municipio comenta acerca de los efectos económicos y el 'daño' que genera a las instituciones formales de agua la operación de actores no autorizados. En su declaración podemos rescatar esta 'disputa' entre no solo organismos operadores sino entre prácticas del orden y de sistemas legales, es decir, entre comunidades imaginadas, para referir el concepto utilizado por Anderson (1983).

El problema es que hay muchas pipas de agua y pozos sin registro o permiso para ser operados. Hay propietarios de pozos que venden agua y hay reventa de agua en pipas. Es complicado en cuanto a normas. Las ventas dentro del mercado negro de agua territarias 30

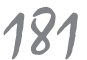


afectan el trabajo de los organismos operadores de agua [formales, descentralizados del municipio]. Los pequeños comités de agua consuetudinarios, las ventas pequeñas y las reventas son ilegales porque estas causan pérdidas millonarias a Ayst. En la comunidad de San Mateo Atenco, por ejemplo, la venta de agua en pipas está prohibida. Sin embargo, AysT seguido vende agua en pipas debido a las limitaiones económicas que hay dentro de esta institución. Ayst usualmente vende [agua] a aquellos asentamientos irregulares o donde esta institución sabe que no habrá agua durante una temporada (entrevista a administrador público municipal, Toluca, junio 2009).

Por su parte, los dueños de los pozos que cubren los requisitos para obtener permisos de extracción también opinan que los vendedores informales no deben tener derecho de acceso al agua puesto que no pagan impuestos ni a Conagua ni a AysT:

Hay muchísimas personas que no pagan por los derechos de agua. Esto no conviene para nada al gobierno [instituciones formales] porque estas personas solo están exprimiéndolo (sic) sin contribuir con nada. Hay muchos vendedores informales y muchos propietarios de pozos no autorizados quienes no tienen permisos para usar el agua. Sin embargo, lo hacen. Alguna vez, alguien vino a verme para ofrecerme a la venta parte de sus derechos de agua, parte de los metros cúbicos a los que tiene acceso. Me pedían millones de pesos, pero yo no sé cómo es que hace eso. Se supone que eso es imposible, no está permi- tido y ellos no deberían vender. Por lo tanto, todo esto representa pérdidas importantes para el gobierno (entrevista a propietario de un pozo, San Francisco, abril 2009).

Los vendedores locales de agua no pagan por los derechos de extracción a Conagua — dueña por definición jurídica de todas las aguas nacionales-, porque no poseen una concesión sobre el agua. A pesar de ello, estos vendedores informales encuentran la manera de tener acceso formal para la extracción de agua de los acuíferos de la región. A su vez, los vendedores de agua a través de pipas, no tienen derechos para gestionar el agua. Sin embargo, sí hacen valer su derecho para excluir a otros usuarios de tener acceso al agua que venden. Esto lo hacen eligiendo a qué usuarios, oficinas o negocios prefieren venderles agua y a quienes no. El criterio de venta generalmente depende de la distancia que deben recorrer con el líquido para hacer llegar el recurso a las casas.

Por el contrario, los comités de agua comunitarios consideran a los vendedores (también informales) como una alterntiva viable para obtener agua en los momentos en que se enfrentan dificultades para proveer el servicio. Los comités de agua categorizan como deudores a aquellos usuarios no autorizados, haciéndoles notar que sin pagar por el derecho de acceso al agua no tienen derechos de uso. A su vez, también los usuarios domésticos no autorizados o los deudores, buscan la forma de accesar al agua potable a través de sus redes sociales o a través de la compra de pipas de agua. 


\section{Legitimidad y reconocimiento de los derechos sobre el agua}

En las comunidades de estudio no existen mecanismos para legitimar a los vendedores informales de agua en pipas, igualmente para las instituciones formales ellos no alcanzan la categoría de usuarios autorizados. Por su parte, los usuarios de agua no autorizados que tienen deudas también encuentran difícil ponerse al corriente en sus pagos por el servicio que administra el comité de su comunidad, puesto que los pagos dependen generalmente de su disposición a pagar y de su capacidad económica que no siempre es ideal. La disposición para pagar normalmente está influenciada por la suficiencia y calidad en el servicio que reciben. Por esta razón, al no haber calidad en el servicio tampoco hay pago por parte de algunos usuarios. Paralelamente, tampoco hay mecanismos para obligar a los usuarios deudores a pagar (morosos), ni mecanismos para legitimar el acceso de los usuarios domésticos al agua (tanto al agua de la comunidad como aquella que obtienen a través de los revendedores).

Bajo estas circunstancias, los actores no autorizados finalmente encuentran mecanismos de adaptación para accesar al agua que les permitirá cubrir necesidades básicas, no obstante las limitantes económicas o geográficas. Asimismo, esto hace que aun existan actores no autorizados que ejercen su 'derecho en acción' para alcanzar los recursos hídricos que necesitan.

La forma en que tanto los miembros de una comunidad como las instituciones informales legitiman y reconocen el derecho de acceso de los actores depende del uso histórico del agua, de los acuerdos generados en asambleas públicas y del pago de los derechos a la autoridad responsable.

De acuerdo con Boelens y Zwarteveen, Roth y Boelens (2005) y Boelens (2008) en un sistema informal hay actores que han ido ganando derechos sobre el agua a través de la mano de obra puesta en la construcción, instalación o mantenimiento de infraestructura; al igual que otros a través de años de negociación sobre el uso histórico del agua.

Años de prácticas tradicionales han hecho que comunidades hídricas como San Francisco o Santiaguito se hayan organizado para usar el agua bajo sus propios modos y tradiciones. Por ejemplo, el agua superficial ha sido utilizada para agricultura de irrigación, mientras que las aguas subterráneas han sido utilizadas para consumo doméstico. ${ }^{5}$

La comunidad de San Francisco tiene derechos históricos para acceder al agua subterránea. Desde la época colonial, esta comunidad, así como sucede en otras de México, han comprometido su mano de obra colectiva para organizar la infraestructura de agua para irrigación y dotación del servicio de agua potable, aunque aún enfrentan carencias fuertes. San Francisco ha mantenido un régimen consuetudinario porque la comunidad se asentó sobre un área rica en agua superficial y subterránea, lo cual le permitió ser autosuficiente. La mayoría de las casas tenían norias tradicionales con las cuales cubrían sus necesidades hídricas. Sin embargo, desde 1970, la
${ }^{5}$ Desde una perspectiva general, esta situación es un reflejo de lo que sucede en algunos países latinoamericanos donde autores han identificado que tanto las aguas superficiales como las subterráneas han sido históricamente distribuidas y utilizadas con fines de irrigación y consumo humano (Beccar, Boelens y Hoogendam, 2002). territarios 30

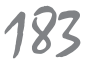


expansión urbana y los planes de desarrollo de México orientados para gobernar el agua han promovido entubar el agua más que continuar con las formas de extracción tradicional. Con ello, los derechos para gobernar el agua y las formas de gestión del servicio de agua potable también han sido modificadas en las comunidades mexicanas. Antes de 1970, los habitantes eran los dueños de los pozos de agua y norias abiertos dentro de su propiedad; por lo tanto, las decisiones para usar, extraer, donar o compartir agua dependían en su totalidad de cada dueño; no tenían que consultar a instituciones formales o informales para acceder al agua, tampoco tenían que solicitar permisos para usar cantidades específicas.

Después de 1970 los derechos de propiedad sufrieron cambios en todo México; los derechos de propiedad del agua fueron nacionalizados y el Estado, a través de la Comisión Nacional del Agua (Conagua), asumió todos los derechos de agua y se nombraron responsables de distribuir y reconocer los derechos de propiedad de las diferentes comunidades.

De tal modo que en la actualidad Conagua es la institución dueña de las aguas que se encuentran dentro del territorio nacional y la responsable de aprobar títulos de concesión y reconocer los derechos de agua de los diferentes usuarios, especialmente los que buscan ser legitimados como propietarios. En este sentido fue que comunidades como Santiaguito, San Francisco y San Mateo fueron reconocidas con derechos de propiedad sobre los pozos de agua que se encuentran dentro de la comunidad. Los ciudadanos, a su vez, han elegido democráticamente, de manera consuetudinaria, a los miembros del comité de aguas que se han hecho cargo de gobernar y gestionar el servicio de agua potable.

En el caso de Santiaguito y San Mateo no poseen derechos históricos para el manejo del agua, pero sí adquieren derechos que son reconocidos por los usos y costumbres de su comunidad. Con estos derechos de propiedad, la comunidad se organiza para gobernar el agua localizada dentro de su territorio, y proveer un servicio legitimado por la ciudadanía y administrado por el comité. Para legitimar los derechos de cada actor, tanto los consumidores como los miembros del comité de aguas han acordado pagar una tarifa mensual, no por el uso del agua - viéndolo como recurso natural-, sino como un servicio que requiere de infraestructura y mantenimiento. La tabla 2 resume la cantidad aprobada por

Tabla 2. Tarifas aprobadas por la comunidad para legitimar el servicio de agua potable

\section{territarias 30} 184

\begin{tabular}{|l|c|c|c|}
\hline \multicolumn{1}{|c|}{ Comunidad } & San Mateo & Santiaguito & San Francisco \\
\hline fa mensual & $\$ 70 \mathrm{MXN}$ & $\$ 50 \mathrm{MXN}$ & $\$ 50 \mathrm{MXN}$ \\
\hline por semana en los que reciben agua potable & 7 y 3 en algunas áreas céntricas & 3 & 1 \\
\hline
\end{tabular}

Fuente: Elaboración a partir de datos obtenidos a través de entrevistas, 2010. 
las comunidades estudiadas para legitimar el acceso de los diferentes usuarios al servicio de agua potable.

El pago por este servicio en cada comunidad varía en función de los acuerdos aceptados y legitimados por los usuarios. El esquema de funcionamiento por costumbre depende de las necesidades de la población y de los acuerdos para reconocer los derechos de propiedad de cada actor en función de su participación dentro de la comunidad y del pago por el agua que consumen.

No obstante los pagos y los acuerdos generados, un sistema consuetudinario se enfrenta a procesos de violación de acuerdos, los cuales generalmente se deben a condiciones económicas, a intereses personales, o por fallas en la infraestructura y las limitantes financieras para componerla en el corto plazo.

Los procesos que definen el servicio de dotación de agua potable que se dan a la comunidad, las subjetividades del proceso, y su legitimidad 'fuera' del Estado, permiten entonces ser comparados con otras subjetividades que tienen lugar en distintas zonas y a diferentes escalas (De Alba, 2012).

La construcción de una comunidad hídrica imaginada se da a partir de las precariedades locales del agua, no obstante que haya abundancia, el problema es continuamente la gestión, la forma de gobierno y cómo se legitiman sus instituciones.

\section{Conclusiones}

Bajo la noción de comunidad hidrica imaginada de Anderson (1983) en este artícu- lo se han estudiado casos de comunidades originarias que han desarrollado procesos identitarios y forma de legitimación colectiva a través de la apropiación del recurso agua. A través de esta noción, ha sido posible observar cómo las comunidades hídricas localizadas en el estado de México, han obtenido mayor empoderamiento (empowerment) sobre el recurso natural y otras capacidades para disputar al Estado la dirección, el control, el manejo, el acceso y el cobro del servicio de agua potable. Esta situación no es exclusiva del Estado de México. Los casos de estudio son representativos de una situación que puede ser encontrada en países colonizados, como lo es el caso de comunidades en México, y latinoamericanos.

La noción de comunidades hídricas imaginadas contribuye a identificar otros procesos alternativos de organización y legitimidad que se contraponen a las miradas oficiales, las cuales ven en este proceso de legitimidad y apropiación una disputa económica por un recurso natural, o una disputa administrativa por un servicio para la población. No obstante, la comunidad no necesariamente las ve en términos económicos o administrativos sino en términos de propiedad y acceso al agua. Estos otros procesos que se llevan a cabo con la ciudadanía, son los procesos identitarios y de legitimación de un colectivo sociopolítico que busca mantener el control del agua sin necesidad de gestionar su distribución y uso ante instituciones formales; ejemplos de este tipo de comunidades hídricas son los casos de las comunidades originarias territarias 30

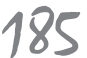


aquí estudiadas. Estas se constituyen por ciudadanos y forman comités de agua para lograr una mejor organización comunitaria. Igualmente, buscan tener una institución (frecuentemente informal) que asuma el reto de prestar un servicio comunitario a través de la organización del servicio de agua potable, su manejo y mantenimiento. De esta forma, la operación y administración del servicio de agua potable por parte de los comités de aguas, da pauta a la generación de formas de construcción de comunidad, en las cuales los habitantes de una comunidad hídrica se asumen participantes dentro de los procesos de legitimación. Este tipo de comunidades no se han encontrado exclusivamente en la zona de estudio, también se tiene conocimiento de ellas en distintas regiones de México, así como en países latinoamericanos. Sin embargo, como casos representativos, en este artículo se tomaron en cuenta tres comunidades del estado de México.

En la opinión de un representante del agua, la responsabilidad de los integrantes del comité es participar temporalmente retribuyendo a la comunidad a través de un servicio.

Lo que nosotros hacemos es un servicio a la comunidad. Nosotros estamos contentos de cooperar porque hay otros años en los que alguien más hace este trabajo. Yo sé que el servicio que proporcionamos como comité de aguas es solo por un año. Durante este año tratamos de dar lo mejor de nosotros, incluso cuando no podemos estar aquí, en la oficina, todo el día... Durante este tiempo es- cuchamos todo acerca de los problemas con el agua, tratamos de solucionar las quejas, recogemos los pagos por el agua y también, tratamos de contactar a los deudores para presionarlos a que paguen sus deudas, y, así, poder obtener más dinero, a pesar de que sabemos que no es posible lograrlo siempre (presidente del comité de aguas de Santiaguito, julio 2010).

El entusiasmo con que inicia un comité de aguas es una característica importante para trabajar a favor de la comunidad, beneficiándola y buscando mejores condiciones en los servicios que este provee $y$, por tanto, en su calidad de vida. No obstante, los comités enfrentan dificultades que los pueden hacer desistir del cargo, abandonarlo o beneficiarse individualmente de los pagos que reciben.

Las principales dificultades que enfrentan los comités de agua a nivel comunitario son: falta de pago por el servicio de agua por parte de todos los usuarios domésticos, teniendo así un déficit en el ingreso con respecto a los gastos por la operación del servicio de agua potable. También se enfrentan, constantemente, al mal manejo de recursos económicos por parte del tesorero, quien aunque es miembro del comité, frecuentemente ha hecho mal manejo de los pagos de los usuarios para beneficiarse. Los miembros del comité de aguas también se enfrentan a la falta de remuneración económica. Es cierto que el puesto temporal que ocupan es un servicio a la comunidad; sin embargo, el no recibir un pago por este servicio hace que busquen tener otro 
empleo remunerado para sostener a su familia, por ello, al manejo, operación y supervisión del servicio de agua, generalmente, destinan tiempos menores.

Otro problema que frecuentemente enfrentan los miembros de los comités de agua es la deslegitimación de su 'puesto' por parte de la ciudadanía. Los usuarios pueden deslegitimar a las autoridades locales en turno y elegir nuevas que estén dispuestos a aportar beneficios comunes. Esto sucede cuando los ciudadanos observan que algún miembro del comité (frecuentemente el tesorero) o todo el equipo de trabajo hace malos manejos de los recursos económicos sin consultarlo con los ciudadanos. En esos casos, la comunidad deslegitima dicha autoridad y elige una nueva. La capacidad de los usuarios de elegir a sus representantes, decidir en función de sus necesidades y legitimar autoridades y decisiones, les da poder para procurar mantener los derechos de propiedad de los recursos hídricos dentro de la comunidad, los derechos de propiedad sobre la infraestructura hidráulica y de los pozos que tradicionalmente le han pertenecido a la comunidad por encontrarse dentro de esta y de la capacidad de autoorganizarse $\mathrm{y}$ administrarse.

Un aporte más de este artículo es que las evidencias empíricas estudiadas han sido entendidas a través de conceptos teóricos tales como: empoderamiento de los miembros comunitarios, comunidades hídricas imaginadas, Estado, instituciones informales y actores comunitarios. A través de estos conceptos este artículo ha presentado un acercamiento a la administración de re- cursos hídricos, en especial del servicio de agua potable por parte de los miembros de comunidades que se autogobiernan para proveer este servicio. Estas comunidades, buscan mantener su autonomía para tener control sobre el agua de su comunidad y así decidir y legitimar su administración, mantenimiento e infraestructura, de tal modo que les permita no depender de las decisiones y legitimidad por parte de las instituciones del Estado. A través del estudio de comunidades que se organizan por costumbre, en el Estado de México, es posible acercarnos a la interpretación de otras comunidades u otros casos de estudio y también a procesos similares, en diferentes contextos, tanto nacionales como internacionales.

Finalmente, como perspectiva teórica, la noción comunidades imaginadas, utilizada aquí para comprender procesos comunitarios, de identidad y de legitimación política, se ha revelado pertinente para el análisis de: 1) comunidades que se encuentran en disputa por este recurso natural; 2) de aquellas comunidades que desarrollan procesos de resistencia social al despojo del mismo, por parte de autoridades locales, estatales o federales; o 3 ) de todas aquellas comunidades originarias que se organizan de forma separada o intentan, por diferentes vías, remplazar al Estado en funciones.

\section{Referencias}

Alexander, J. C., (2003). The meanings of social life. New York: Oxford University Press. territarias 30

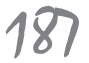


Anderson, B. (1983). Imagined communities: Reflections on the origins and spread of nationalism. London: Verso.

Appuradai, A. (2003). Sovereignty without territoriality: Notes for a postnational geography. En S. Low y D. LawrenceZuniga (eds). The Anthropology of Space and Place: Locating Culture (pp. 337 49). Boston: Blackwell Publishing.

Archer, M. S. (1996). Culture and agen$c y$. New York: Cambridge University Press.

Beccar, L., Boelens, R. y Hoogendam, P. (2002). Water rights and collective action in community irrigation. En R. Boelens y P. Hoogendam (eds). Water rights and empowerment (pp. 1-21). Assen: Van Gorcum.

Becerril-Tinoco, C. A. (2012). Governance of the drinking water supply service: a case study of three Mexican communities (Tesis doctoral, Universidad de East Anglia, Norwich, Reino Unido). Recuperado de https://ueaeprints.uea. ac.uk/42413/

Boelens, R. A. (2008) The rules of the game and the game of the rules: normalization and resistance in Andean water control. Wageningen: Wageningen University.

Boelens, R. y Zwarteveen, M. (2005). Prices and politics in Andean water reforms. Development and Change, 36(4), 735758.

Davis, D. (2010). Irregular armed forces, shifting patternsof commitment, and fragmented sovereignty in the develo-
De Alba, F. (abril, 2012). México y los imaginarios de la acción política 'fuera' del Estado ¿Cómo analizar la protesta desde sus desplazamientos e identidades locales? European Review of Latin American and Caribbean Studies, (92), 39-58.

Devetak, R. y Higgott, R. (mayo, 1999). Justice unbound? Globalisation, states, and the transformation of the social bond [Working Paper 29/99]. University of Warwick.

Helmke, G. y Levitsky, S. (2004). Informal institutions and comparative politics: a research agenda. Perspectives on politics, 2(4), 725-740.

Linklater, A. (1993). The transformation of political community: Ethical foundations of the Post-Westphalian Era. Cambridge: Cambridge University Press.

Lynch, O. J. y Talbott (1995). Balancing acts: community-based forest management and national law in Asia and the Pacific. Washington: World Resources Institute.

Matsinhe, N. P., Juízo, D., Macheve, B. y Santos, C. D. (2008). Regulation of formal and informal water service providers in peri-urban areas of Maputo, Mozambique. Physics and Chemistry of the Earth, Parts $A / B / C, 33(8-13)$, 841-849.

Ostrom, E. (2001). The puzzle of counterproductive property rights reforms: a conceptual analysis. En A. De Janvry, G. Gordillo, J. P. Platteau y E. Sadoulet (eds.). Access to land rural poverty and public action. New York: Oxford University Press. 
Pahl-Wostl, C. (2009). A conceptual framework for analyzing adaptive capacity and multi-level learning processes in resource governance regimes. Global Environmental Change, 19(3), 354-365.

Sassen, S. (2007). Territory, authority, rights: From medieval to global assemblages. Princeton: Princeton University Press.

Schlager, E. y Ostrom, E. (1992). Property rights regimes and natural resources: a conceptual analysis. Land economics, 68(3), 249-262.

Von Benda-Beckmann, F. (1995). Anthropological approaches to property law and economics. European journal of law and economics, 2(4), 309-336.
Von Benda-Beckmann, F., Von Benda-Beckmann, K. y Spiertz J. (1998). Equity and legal pluralism: taking customary law into account in natural resource policies. R. Boelens y G. Dávila (eds.). Searching for equity. Conceptions of jus tice and equity in peasant irrigation. Assen: Van Gorcum.

Zwarteveen, M., Roth, D. y Boelens, R. A. (2005). Water rights and legal pluralism. Beyond analysis and recognition. En D. Roth, R. A. Boelens y M. Zwarteveen (eds.). Liquid relations. Contested water rights and legal complexity (254-278). New Brunswick: Rutgers University Press. 
